\title{
Performance of 3-D Architecture Silicon Sensors After Intense Proton Irradiation
}

\author{
Sherwood I. Parker and Christopher J. Kenney
}

\begin{abstract}
Silicon detectors with a three-dimensional architecture, in which the $n$ - and p-electrodes penetrate through the entire substrate, have been successfully fabricated. The electrodes can be separated from each other by distances that are less than the substrate thickness, allowing short collection paths, low depletion voltages, and large current signals from rapid charge collection. While no special hardening steps were taken in this initial fabrication run, these features of three-dimensional architectures produce an intrinsic resistance to the effects of radiation damage. Some performance measurements are given for detectors that are fully depleted and working after exposures to proton beams with doses equivalent to that from slightly more than ten years at the B-layer radius (50 $\mathbf{m m}$ ) in the planned Atlas detector at the Large Hadron Collider at CERN.
\end{abstract}

Index Terms-Detectors, pin diodes, radiation hardness, semiconductor detectors, silicon detectors, three-dimensional electrodes.

\section{INTRODUCTION}

INCE the first custom VLSI chip was developed for the $\checkmark$ readout of silicon strip detectors [1], both the chips and the detectors have been fabricated by planar technology methods. With the precision provided by small strip spacing and the compact readout provided by the VLSI chips, such detectors have played an increasingly important role in tracking at collider detectors.

The coming generation of high-luminosity colliders will subject their innermost detector elements to extreme levels of radiation. Silicon detectors are subject to a number of damage sources.

1) Ionization-caused charging of their surface oxide passivating layers can attract mobile substrate charges. These in turn may short out adjacent electrodes or increase their interelectrode capacitance [2]-[4]. This charging has been seen to saturate at a value of 2.5 $\times 10^{12}$ charges per $\mathrm{cm}^{2}$ for a 500 -nm-thick oxide, but with no electric field applied during irradiation [5]. The same paper reported effects attributed to continued charging in the similar oxide of a gated diode (but now subject to an electric field) at fluence values more than five times as high, not an unreasonable result with an electric field driving charge to the surface.

Manuscript received August 16, 2000; revised May 17, 2001 and June 11, 2001. This work was supported by the U.S. Department of Energy under Grant DE-FG03-94ER40833 and made use of the National Nanofabrication Users Network facilities supported by the National Science Foundation under Award ECS-9731294.

S. I. Parker is with Lawrence Berkeley Laboratory, Berkeley, CA 94720 USA (e-mail: sher@slac.stanford.edu).

C. J. Kenney is with the University of Hawaii, Honolulu HI 96822 USA

Publisher Item Identifier S 0018-9499(01)09028-1.

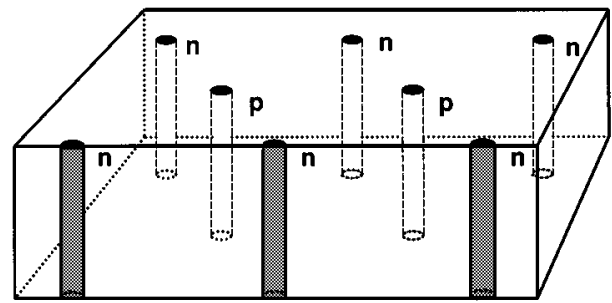

Fig. 1. Schematic, three-dimensional view of part of a sensor with 3-D electrodes penetrating through the substrate. The front border of the figure is drawn through the center of three electrodes.

2) Damage to the crystal lattice produces defects that are negatively charged when the silicon is depleted, causing lightly doped n- and p-type silicon to develop a high depletion voltage of the same sign as that of p-type silicon [6]-[11]. While some of this damage can anneal out, a temperature-dependent reverse annealing can cause a major increase in such damage [12]-[15]. Maintaining the detectors continuously at -5 to $-10{ }^{\circ} \mathrm{C}$ prevents this reverse annealing, but if the cooling is ever lost for more than a few days, the damage will be irreversible. A model for this bulk damage, involving divacancies and divacancy-oxygen defects, $\mathrm{V}_{2} \mathrm{O}$, has been developed, which explains effective doping changes, leakage current increases, and the leakage current temperature dependence for ${ }^{60} \mathrm{Co}$ gamma irradiation [16]. Such detailed agreement is not found for hadron interactions, which create dense damage clusters that are not yet understood in detail.

3) The charge carrier lifetime decreases, also from bulk damage [17]-[19]. (In addition, the lifetime was found to vary with bias voltage [20].)

Recent work has shown that the incorporation of about $10^{17}$ oxygen atoms per $\mathrm{cm}^{3}$ increases the fluence, by approximately a factor of two, for a given increase in those negatively charged defects [21]-[24]. This beneficial effect is not present with neutron irradiation, indicating that it may be the damage from the less-dense Coulomb interactions that is being reduced. The incorporation of oxygen also does not seem to reduce leakage currents [25].

This paper describes the first radiation-damage test results for a new form of silicon detector, which has a three-dimensional (3-D) array of $n$ - and p-electrodes perpendicular to the surface and which penetrate the entire thickness of the detector [26]-[29]. Fig. 1 shows a schematic view of such a detector. The low depletion voltages and short collection paths for ionization charges made possible with 3-D technology result in an intrinsic 
resistance to the effects of bulk silicon damage. Attracted mobile substrate charges [point 1) above] can be immobilized with field implants or kept from accumulating with a proposed new type of field plate that does not significantly increase the effective interelectrode capacitance [30].

Calculated properties, fabrication steps, and initial results from 3-D detectors, made with high-purity, 100, p-type silicon, have been described in earlier publications [26]-[29]. Two sets of narrow cylindrical holes are etched through each wafer, using a plasma etcher designed for the rapidly developing field of micromachining. After each set is etched, it is filled with nor p-doped polycrystalline silicon. This is done by exposing the wafers to silane gas $\left(\mathrm{SiH}_{4}\right)$. Some molecules stick, lose their hydrogen atoms, and migrate along the surface to join with other silicon atoms, eventually forming a $2-\mu \mathrm{m}$-thick layer of polycrystalline silicon.

The pressure is kept low so the silane molecules have a mean free path long compared with the hole dimensions. The temperature is also kept low enough so that the probability of a molecule sticking after a surface collision is low. Under these conditions, when a molecule does remain on the surface of the hole, it is almost as likely to be at the bottom as the top, thus producing a conformal layer. This is followed by the deposition of appropriate dopant gases such as $\mathrm{P}_{2} \mathrm{O}_{5}$ and $\mathrm{B}_{2} \mathrm{O}_{3}$ and by a final deposition of polycrystalline silicon, which eventually fills the hole. Heating then lets the dopant diffuse into the single-crystal silicon, where it can make a low-leakage diode junction.

\section{DETECTORS}

The detectors were $121 \mu \mathrm{m}$ thick and were diced, but remained bonded to a substrate left over from a support wafer that prevented cracking during fabrication. They had alternating rows of $\mathrm{n}$ - and p-electrodes with diameters slightly larger than the $17-\mu \mathrm{m}$-diameter holes, due to the dopant diffusion. The n-to-n and p- to-p row pitch was either 100 or $200 \mu \mathrm{m}$. The n-rows were placed halfway between the p-rows and with their electrodes shifted 67 or $50 \mu \mathrm{m}$, respectively, along the row direction, so they were halfway between the p-electrode positions in that direction also. Most electrodes were bused together along each row and brought out to probe card contacts along two opposite edges, as shown in Fig. 2. Several, also visible in Fig. 2, were left isolated, so they could be contacted individually with low-capacitance probes. Three bands of guard electrodes, alternately p-type, n-type, and p-type, surrounded the array. (In future devices, these may be replaced with an active edge just outside the array [27], [29].)

For this first-ever fabrication run of 3-D devices, we decided to keep the steps simple and did not use either field implants or field plates to prevent the accumulation of electrons under the passivating surface oxide. With the strong horizontal repulsive forces from the p-type electrodes, these electrons did not seriously interfere with data collection from the $\mathrm{p}$ electrodes. We also did not add oxygen to the silicon wafers, so any benefit resulting from that should further increase the radiation hardness of these devices.

\section{IRRADIATION}

Seven detectors were tested and then packaged in polyimide envelopes and irradiated with 1,2 , or $10 \times 10^{14}, 55$ $\mathrm{MeV}$ protons per $\mathrm{cm}^{2}$ at the LBL 88 -in cyclotron. The fluence was measured with an ionization chamber divided into a series of concentric rings. Each fed an integrating amplifier, which reset at a preset charge level. The number of resets was then counted to determine the total fluence. The ring area was normalized out, and so all ring numbers normally increased at close to the same rate for uniform beams. During subsequent runs, this number, for one ring or another, was observed to make a small jump to a higher value and then continue its uniform climb. The source of these jumps is not yet known, but they are now easily recognized and removed from the data. The numbers were not tracked at the time of our run, and 5-10\% variations in the ratio of the two ring values that were recorded for the three runs could have been due to such jumps rather than to beam uniformity variations.

Two other detectors were irradiated with $5.19 \times 10^{14}, 24$ $\mathrm{GeV}$ protons per $\mathrm{cm}^{2}$ at the CERN proton synchrotron. For this first irradiation, the detectors were unpowered. After irradiation, all detectors were kept cooled, mostly at $-7^{\circ} \mathrm{C}$, except during measurements, when they were at about $21{ }^{\circ} \mathrm{C}$. The total time not cooled is less than $10 \mathrm{~h}$ for any detector, so they have not yet been annealed to the point of minimum damage.

A number of papers have been published comparing the ratio between particle flux and changes in leakage currents and depletion voltages as a function of particle type and energy [31]-[38]. These have generally found that the increase in leakage currents and depletion voltages scales with the nonionizing energy loss (NIEL), which, coming from nuclear collisions, is presumably proportional to the number of damage centers responsible for the increases. Thus, measurements taken in one beam must be normalized, using the NIEL factors, to the irradiation expected during the experiment. Most of the hadron flux through the inner silicon layers is expected to come from pions from the delta 33 resonance with a peak around $200 \mathrm{MeV}$ and with much of the flux between 100 and $500 \mathrm{MeV}$ [39]. Over this range, the NIEL, normalized to that of $1 \mathrm{MeV}$ neutrons (95 $\mathrm{MeVmb}$ ), ranges from 0.70 (at $100 \mathrm{MeV}$ ) to 0.94 (200 MeV) to $0.55(500 \mathrm{MeV})$ [32]. The corresponding normalized ratios for protons at $55 \mathrm{MeV}$ and $24 \mathrm{GeV}$ are 1.715 and $0.51 .1^{1}$ Using 0.8 as a rough estimate for the LHC pion NIEL, the equivalent pion fluence for the four irradiation exposures-1, 2, and $10 \times 10^{14}, 55 \mathrm{MeV}$ protons per $\mathrm{cm}^{2}$, and $5.19 \times 10^{14}, 24 \mathrm{GeV}$ protons per $\mathrm{cm}^{2}$ - are 2.1, 4.3, 21, and 3.3 $\times 10^{14}$ pions per $\mathrm{cm}^{2}$.

The third fluence value, from the $10^{15}, 55$ $\mathrm{MeV}$ protons per $\mathrm{cm}^{2}$ run, which was limited simply by the available cyclotron time, is equivalent to that expected in slightly more than $10 \mathrm{y}$ at the $50-\mathrm{mm}$ radius of the planned B-layer. The smaller depletion voltage changes when silicon contains added oxygen indicates the damage per unit NIEL depends on material type. The future use of such silicon in

\footnotetext{
${ }^{1}$ Rather than attempting to read from small published graphs, we used tabulated values from tables prepared by A. Vasilescu and G. Lindström, http://sesam.desy.de/ gunnar/Si-dfuncs-general.html.
} 
3-D detectors could further increase the radiation tolerance of inner layer detectors.

No equipment was available, at the time of these runs, to apply a voltage bias to the detectors. Some recent work indicates that biased detectors may have a depletion voltage increase double that of unbiased ones [40], [41]. Both neutrons and pions showed closely similar effects. No data were given for any variation with the value of the bias voltage, so it is not known if the effect would be different with the small fields present in 3-D detectors.

\section{MEASUREMENTS}

Measurements were made at $21{ }^{\circ} \mathrm{C}$ with unirradiated 100and $200-\mu \mathrm{m}$ pitch detectors and with irradiated $200-\mu \mathrm{m}$ ones at the four different exposures, as well as for a $100-\mu \mathrm{m}$ pitch detector at the $1 \times 10^{15}$ exposure. Before and after these measurements, the detectors were kept at $-7{ }^{\circ} \mathrm{C}$, with the total time at $21{ }^{\circ} \mathrm{C}$ kept short compared to that required for full beneficial annealing. (A system to flood the detector with dry nitrogen has now been installed and should allow future measurements to be made below room temperature without water condensing on the detector surfaces. Work is now under way on the fabrication of the next group of 3-D detectors, which will have such measurements.)

The detectors used for these measurements had all their $\mathrm{p}$ - and n-electrodes tied to the two aluminum voltage busses, with the exception of several floating electrodes, which were well separated from each other. These were then probed directly with a $12 \mathrm{C}$ picoprobe, which had an input impedance of $1 \mathrm{M} \Omega$ in parallel with $0.1 \mathrm{pF}^{2}$ The probe both supplied the ground bias voltage and read out the signal. Only p-electrode data were used since, at low bias voltages, the n-electrodes in unirradiated detectors were shorted together by a surface electron layer. This layer, induced by the positive oxide interface charge, would normally be removed in detectors with field plates [30] or with an adequate field implant.

Both leakage currents and pulse heights from infrared light were measured as a function of applied voltage. An 820-nm infrared diode was placed on one of the eyepieces of the probe station microscope, using a 10x objective. This produced a reduced and intensified beam, which had a 1/e-penetration distance of about $13.5 \mu \mathrm{m}$ in the silicon. The spot size was larger than the cell being measured, but smaller than the full detector. The average of the signal voltage from 50 light pulses, each 920 ns long and repeated every $8.34 \mathrm{~ms}$, was measured as a function of applied voltage using a digital oscilloscope. (The $920 \mathrm{~ns}$ duration of this signal was set, not by the expected nanosecond response time of the sensor [26], but by the limited light output of the light-emitting diode.) A plateau in signal pulse height, with bias voltage increase, was an indication that full depletion - where there was no further gain in sensitive volume-had been reached. It was also an indication of the absence of a significant loss of charge due to capture, since higher applied voltages reduce the drift time.

We also measured the decrease with time of the integrated signal on one of the isolated electrodes after the end of the light pulse, again using the $12 \mathrm{C}$ picoprobe. Like-type electrodes were

${ }^{2}$ The $12 \mathrm{C}$ picoprobe is made by GGB Industries, Naples, FL 34104 USA.

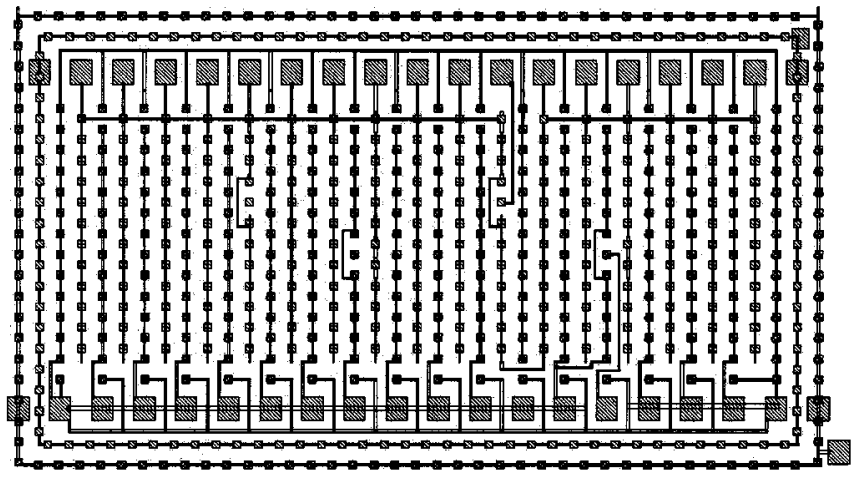

Fig. 2. Metal layer layout for a detector with columns of n-electrodes alternating with columns of p-electrodes. The p-electrodes are shifted $67 \mu \mathrm{m}$ in the column direction (one-half the electrode spacing in that direction). With the exception of several isolated electrodes, all $\mathrm{n}$ - and all $\mathrm{p}$-array electrodes are connected, so two leads from a power supply will energize the entire array. Three bands of guard fences (respectively, p-type, n-type, and p-type) surround the array. In future devices, these may be replaced with an active edge just outside of the detector array.

kept at ground, so except for the small departure from ground due to the signal voltage, the field around the probed electrode was the same as that around the other like-type ones. The electrode capacitance $C_{\mathrm{e}}$ could then be found from

$$
C_{\mathrm{e}}=\left(\frac{\tau}{R_{\mathrm{p}}}\right)-C_{\mathrm{p}}
$$

where

$\tau \quad R C$ time constant of the falling pulse;

$R_{\mathrm{p}} \quad$ input resistance;

$C_{\mathrm{p}} \quad$ capacitance of the probe.

Finally, leakage currents were also recorded for the 200- $\mu \mathrm{m}$ pitch detectors at $21^{\circ} \mathrm{C}$. The $100-\mu \mathrm{m}$ pitch detector, which was exposed to $1 \times 10^{15}$ protons per $\mathrm{cm}^{2}$, had a heat sink compound containing zinc oxide applied for a series of reduced-temperature measurements. Some of the compound spread to the top of the chip, where a galvanic reaction involving condensation moisture caused some surface damage. This resulted in increased leakage current, though not high enough to interfere with the infrared detection tests.

\section{Depletion Voltages}

Fig. 3 shows infrared light-emitting diode signal pulse heights, as a function of applied voltage, on 200- $\mu \mathrm{m}$ n-to-n pitch detectors without irradiation and with all four levels of irradiation. The stability of the voltage plateau can be seen by comparing the shapes of the two sets of points in Fig. 3(c) at $2 \times 10^{14}$ protons per $\mathrm{cm}^{2}$ taken one week apart. The value of the diode light intensity, while constant for each run, varied between them due to differences in setup such as the source position and microscope focus, so only the values of the applied voltages and the associated voltage plateau values have an absolute significance. (Similar results were found for the two sets of points at $1 \times 10^{14}$ protons per $\mathrm{cm}^{2}$ also taken one week apart.) Since we did not want to spend too much time at room temperature, individual bias voltages were changed without waiting the several minutes required at each value for the bias charge distribution and new signal level to settle fully. The 


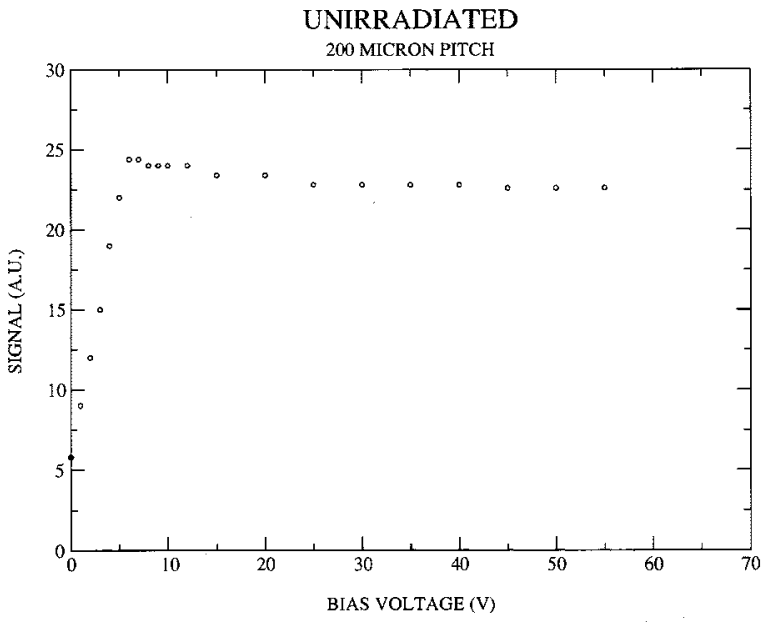

(a)

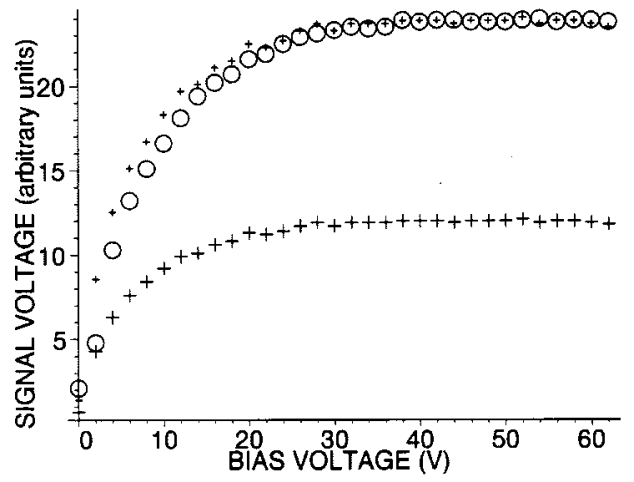

(c)

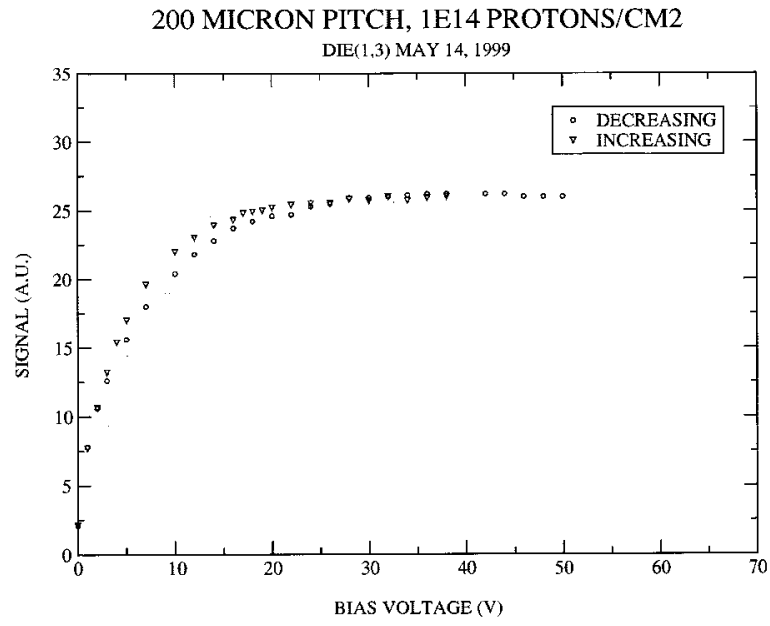

(b)

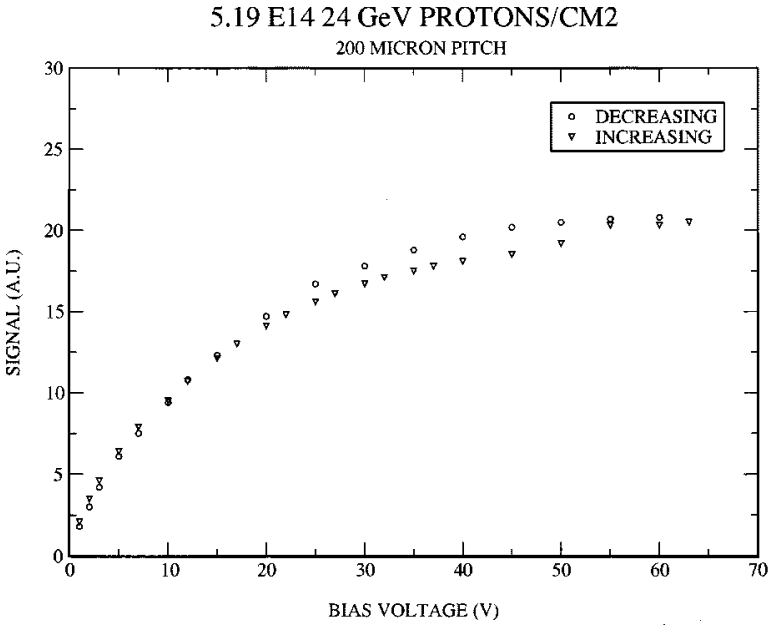

(d)

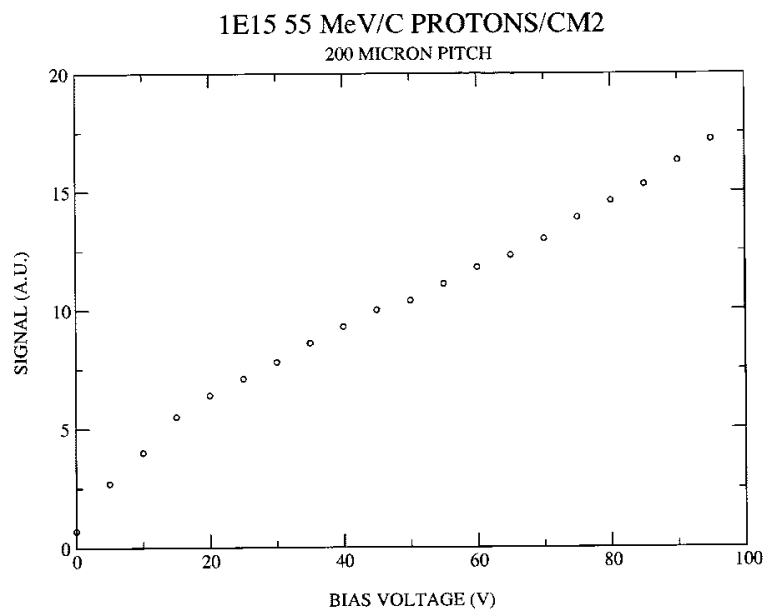

(e)

Fig. 3. Infrared light-emitting diode signal pulse heights as a function of voltage applied to the $200-\mu \mathrm{m}$ (n-n or p-p) pitch detector. The value of the diode light intensity, while constant within each plot, varies from plot to plot, so only the values of the applied voltages and the associated voltage plateau values have an absolute significance. Plots are shown for (a) an unirradiated detector and four detectors irradiated with (b) $1 \times 10^{14} 55 \mathrm{MeV}^{2}$ protons per $\mathrm{cm}^{2}$, (c) 2 $\times 10^{14} 55 \mathrm{MeV}$ protons per $\mathrm{cm}^{2}$, (d) $5.19 \times 10^{14} 24 \mathrm{GeV}$ protons per $\mathrm{cm}^{2}$, and (e) $1 \times 10^{15} 55 \mathrm{MeV}$ protons per $\mathrm{cm}^{2}$. Fig. 3(c) shows values for two runs (crosses and circles) separated by one week and (small crosses) with the earlier run normalized to the later. No plateau was found with the detector exposed to 1 $\times 10^{15} 55 \mathrm{MeV}$ protons per $\mathrm{cm}^{2}$, before the leakage current became excessive.

error this causes in depletion voltage is not large, as can be seen from the difference in points of Figs. 3(b), 3(d), and 4(b), taken while the voltage was being increased and, later, decreased. All but the detector exposed to $1 \times 10^{15}$ protons per $\mathrm{cm}^{2}$ reached the signal plateau value before the leakage current started to approach breakdown values. 
100 MICRON PITCH, PRE IRRADIATION

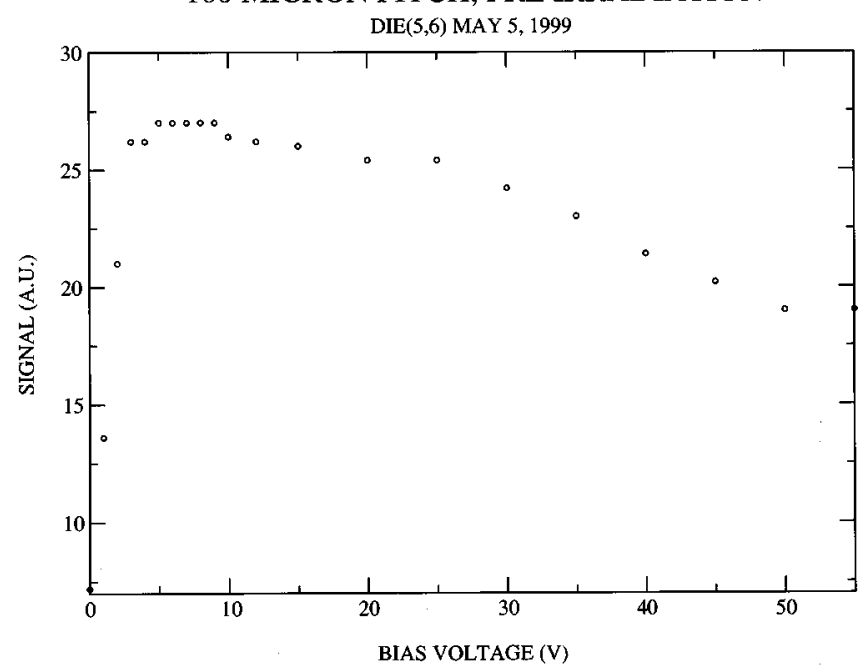

(a)

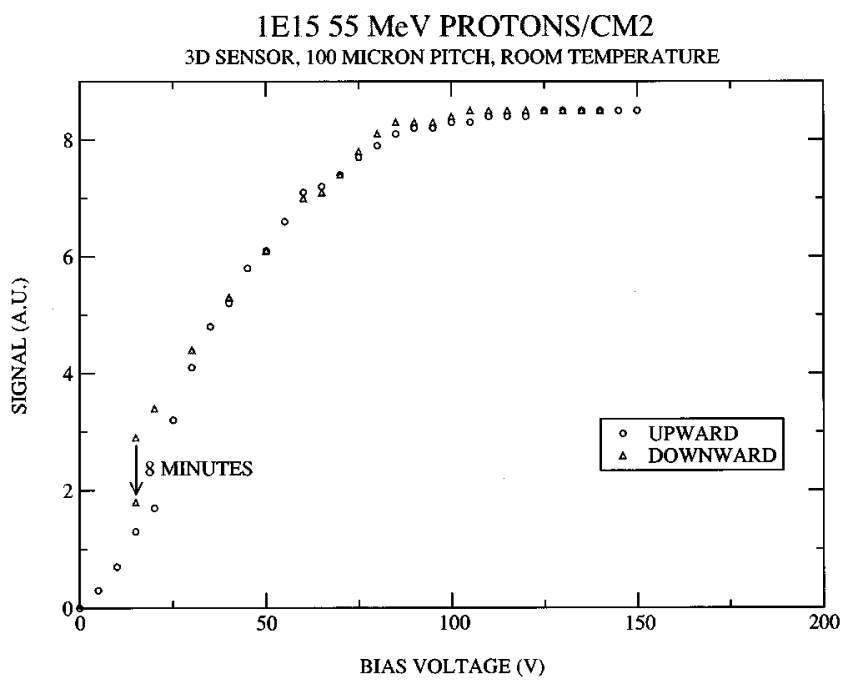

(b)

Fig. 4. Infrared light-emitting diode signal pulse heights as a function of voltage applied to a $100-\mu \mathrm{m}$ pitch detector. Two plots are shown, one before and one after irradiation with $1 \times 10^{15} 55 \mathrm{MeV}$ protons per $\mathrm{cm}^{2}$. The $100-\mu \mathrm{m}$ pitch detector has a plateau between 105 and $150 \mathrm{~V}$.

When the bias voltage is increased, there is a rapid initial rise in signal voltage from the unirradiated sensors [Figs. 3(a) and 4(a)], then a slow decrease. The decrease at larger voltages is due to the attraction of the ionization charge to the metal line connecting all the electrodes in the column other than the one being probed. (An example of such a line can be seen somewhat left of center in Fig. 2.) Infrared-generated charge tends to be made in the upper part of the sensor, and some of it can follow the line to the adjacent p-type electrodes connected to it and bypass the probed electrode. Irradiated sensors do not show this effect, as the radiation-induced positive oxide charge compensates the metal line charge.

Fig. 4(b) shows the signal from a 100- $\mu \mathrm{m}$ sensor exposed to $1 \times 10^{15} 55 \mathrm{MeV}$ protons per $\mathrm{cm}^{2}$. With this reduced pitch, the irradiated detector shows a clear $45-\mathrm{V}$-wide plateau starting at $105 \mathrm{~V}$. An example of the settling time required for belowdepletion bias voltages can be seen from the two points taken at $15 \mathrm{~V}$, separated by $8 \mathrm{~min}$, while the voltage was being reduced.
The plateaus in the charge collection signals shown in Figs. 3 and 4, taken with infrared illumination covering an area that is significantly larger than the cell being probed, indicates both that the depletion voltage has been reached and that the above-depletion electric fields are large enough so capture of charge carriers is not large, despite the radiation damage. Unlike two-dimensional single-sided strip sensors with the diode junction at the strips, where the border between depleted and undepleted silicon covers nearly the full area of the sensor as the bias approaches that for full depletion, the increase in depleted volume in 3-D sensors slows down as the voltage approaches full depletion, since the border shrinks to an ever-smaller cylinder around each ohmic junction. This causes some imprecision (in the 10-20\% range) in determining the depletion voltage, but since it is usually the overall charge collection efficiency itself that is of experimental significance, this should not be a serious problem.

The values for the leakage current and capacitance, given in the next two sections, also show plateaus as the depletion voltage is reached and these values agree, again with a similar uncertainty, with those derived from the signal pulse heights. The increase of leakage currents with increased fields adds an additional error for heavily irradiated samples, and the release of charge carriers from traps in these samples also affects the capacitance determination. But again with these irradiated samples, now with errors somewhat larger than $20 \%$, the values for depletion voltages agree.

\section{LEAKAGE CURRENTS}

Fig. 5 shows room-temperature leakage currents for the same $200-\mu \mathrm{m}$ pitch detectors without irradiation and with all four levels of irradiation. The spike in leakage current for the unirradiated sensor at below-depletion voltages shown in Fig. 5(a) is similar to those in [27, Figs. 15 and 16]. It may be due to the conducting channel of undepleted silicon that runs from the sensor edges, past the guard electrodes, which are not yet at full voltage, to the signal electrodes. It would not be visible against the far higher levels of leakage currents in irradiated sensors. It would also not be expected to be present in active-edge sensors. (See [27, Fig. 12(b)].)

It is generally observed that $I_{\mathrm{rad}}$, the radiation induced leakage current, is given by

$$
I_{\mathrm{rad}}=\alpha \Phi v
$$

where

$\Phi \quad=$ radiation fluence in particles per $\mathrm{cm}^{2}$;

$v=$ depleted volume in $\mathrm{cm}^{3}(0.0121 \times 0.36 \times$ $0.195 \mathrm{~cm}^{3}=8.49 \times 10^{-4} \mathrm{~cm}^{3}$ for $200-\mu \mathrm{m}$ pitch devices);

$\alpha=$ proportionality constant.

The current is measured at the voltage where full depletion is first reached. Leakage currents present before irradiation would, in principle, add a constant term, but they are in the few nanoampere range, about a thousand times smaller than these currents.

Table I shows the depletion voltages, leakage currents, and values of $\alpha$ found for the various runs. To permit comparison of these values of $\alpha$ with others, the $55 \mathrm{MeV}(24 \mathrm{GeV}$ ) proton 


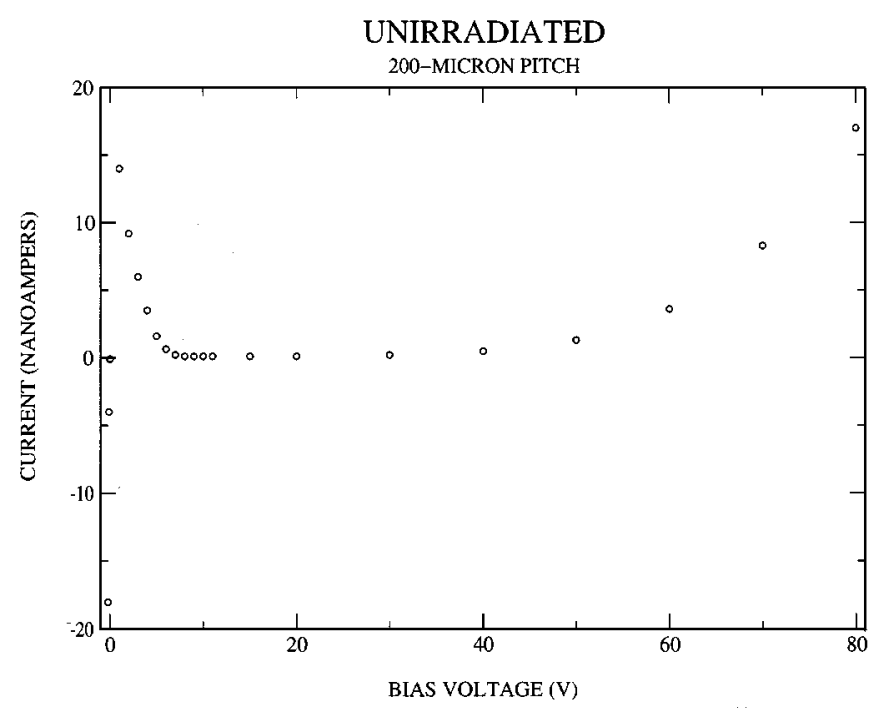

(a)

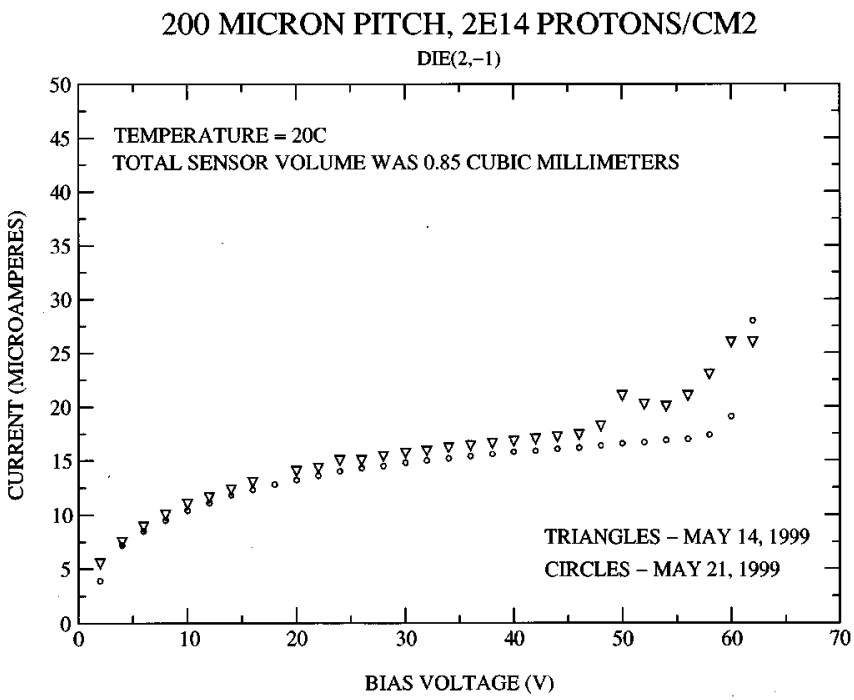

(c)
200 MICRON PITCH, 1E14 55 MeV PROTONS/CM2 TEMPERATURE $=21 \mathrm{C}$

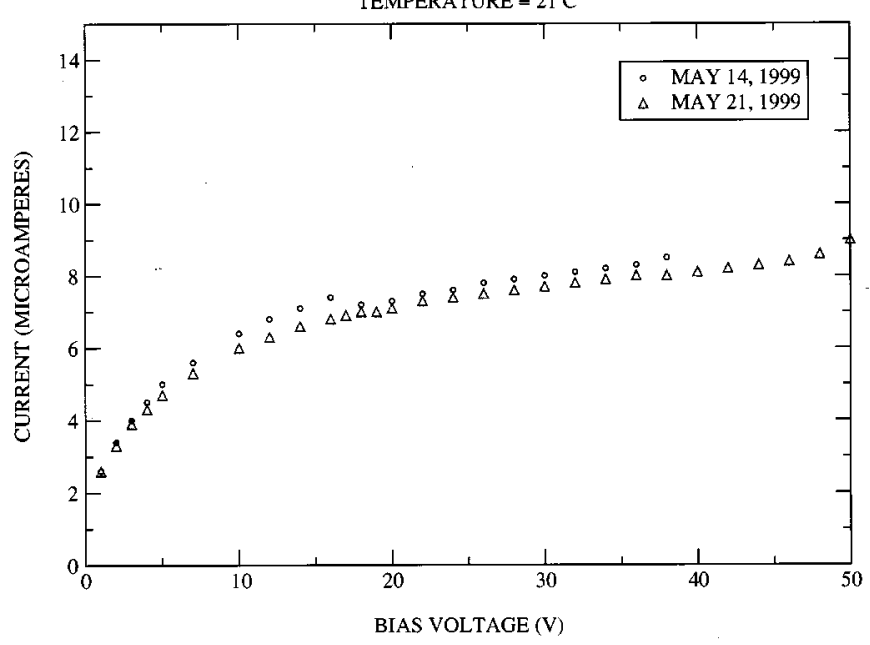

(b)

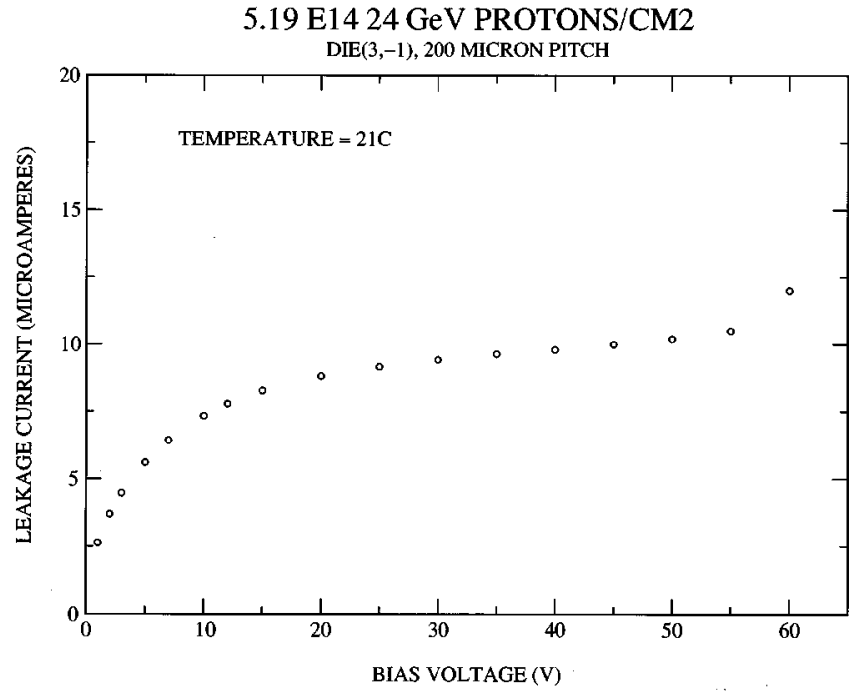

(d)

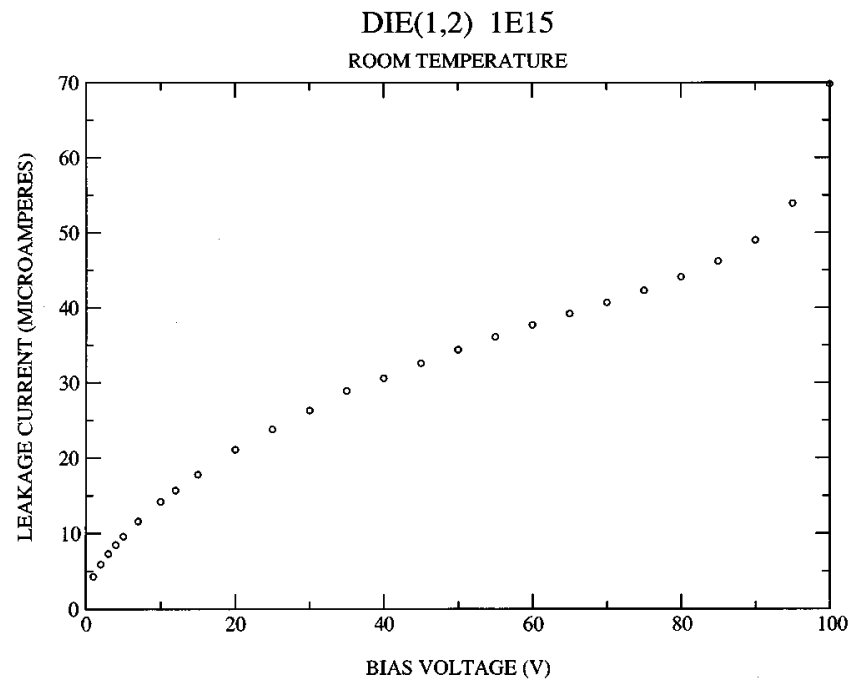

(e)

Fig. 5. Room-temperature leakage currents for $200-\mu \mathrm{m}$ pitch detectors without irradiation and with all four levels of irradiation. 
TABLE I

Depletion Voltages, Room-Temperature Leakage CurRents, Radiation Fluence Proportionality Constant, $\alpha 200-\mu \mathrm{m}$ Pitch $(0.849$ $\left.\mathrm{mm}^{3}\right), 100-\mu \mathrm{m}$ PITCH DETECTORS

(1) see discussion at end of Section V.

(2) variations in sensor-to-sensor values indicate measurement errors are in the $10 \%$ range

(3) range of currents for 15 non-irradiated devices; average $=0.45 \mathrm{nA}(200 \mu \mathrm{m}), 0.26 \mathrm{nA}(100 \mu \mathrm{m})$

(4) room-temperature leakage current increase prevents full depletion

(5) surface damage prevents valid bulk leakage current measurement

\begin{tabular}{l|l|c|c|c|c|c}
\hline Pitch & $\mathrm{E}_{\text {proton }}$ & $\begin{array}{c}\text { Proton fluence } \\
\left(\mathrm{cm}^{-2}\right)\end{array}$ & $\begin{array}{c}\text { Equivalent pion } \\
\text { fluence }\left(\mathrm{cm}^{-2}\right)\end{array}$ & $\begin{array}{c}V_{\text {depletion }} \\
(1)\end{array}$ & $\begin{array}{c}\text { Current } \\
(2)\end{array}$ & $\begin{array}{c}\alpha(\mathrm{A} / \mathrm{cm}) \\
(2)\end{array}$ \\
\hline $200 \mu \mathrm{m}$ & -- & None & None & $6-8 \mathrm{~V}$ & $0.2-1.0 \mathrm{nA}(3)$ & - \\
\hline $200 \mu \mathrm{m}$ & $55 \mathrm{MeV}$ & $1 \times 10^{14}$ & $2.15 \times 10^{14}$ & $20 \mathrm{~V}$ & $7.1 \mu \mathrm{A}$ & $4.9 \times 10^{-17}$ \\
\hline $200 \mu \mathrm{m}$ & $55 \mathrm{MeV}$ & $2 \times \times 10^{14}$ & $4.3 \times 10^{14}$ & $32 \mathrm{~V}$ & $15 \mu \mathrm{A}$ & $5.1 \times 10^{-17}$ \\
\hline $200 \mu \mathrm{m}$ & $55 \mathrm{MeV}$ & $10 \times 10^{14}$ & $21.4 \times 10^{14}$ & $(4)$ & -- & -- \\
\hline $200 \mu \mathrm{m}$ & $24 \mathrm{GeV}$ & $5.19 \times 10^{14}$ & $3.3 \times 10^{14}$ & $50 \mathrm{~V}$ & $10 \mu \mathrm{A}$ & $4.4 \times 10^{-17}$ \\
\hline $100 \mu \mathrm{m}$ & -- & None & None & $5 \mathrm{~V}$ & $0.2-0.5 \mathrm{nA}$ & -- \\
\hline $100 \mu \mathrm{m}$ & $55 \mathrm{MeV}$ & $10 \times 10^{14}$ & $21.4 \times 10^{14}$ & $105 \mathrm{~V}$ & $(5)$ & - \\
\hline
\end{tabular}

fluence given there should be multiplied by 1.715 (0.51) to convert it to the equivalent in $1 \mathrm{MeV}$ neutrons $/ \mathrm{cm}^{2}$. No values are given for the $200-\mu \mathrm{m}$ pitch detector at $10^{15}$ protons $/ \mathrm{cm}^{2}$, since the room-temperature leakage current was too large to permit depletion. While the leakage current proportionality factor $\alpha$ might be expected to have a reasonably constant value at any given measuring temperature, the dependence of the depletion voltage can be more complicated. An initial exponential decrease with fluence has been reported, possibly from the removal from their lattice positions, of some of the initial dopant atoms. Both annealing and reverse annealing occur with different temperature and time dependence and can greatly change the depletion voltage.

\section{Capacitance Measurements}

Fig. 6 shows the decay of the infrared-induced pulse on an isolated electrode of an unirradiated $200-\mu \mathrm{m}$ pitch detector with a $10-\mathrm{V}$ bias voltage. The electrode is grounded through the 1-M $\Omega$ input impedance of a picoprobe, as mentioned in Section IV. Zero time is when the pulse to the light-emitting diode is turned off. After the light emission ends and the charge is collected, the signal height accurately follows an exponential with a time constant of 285 ns. Fig. 7 shows similar decay curves for a range of bias voltages from 0 to $60 \mathrm{~V}$. Fig. 8 shows the decay times derived from Fig. 7. Averaging the values for 10,20 , and $60 \mathrm{~V}$, (1) gives a capacitance of $0.19 \mathrm{pF}$.

Figs. 9 and 10 show similar sets of signal decay curves for detectors irradiated with 1 and $2 \times 10^{14}, 55 \mathrm{MeV}$ protons per $\mathrm{cm}^{2}$. In contrast with the curves for unirradiated detectors, there is now a high tail at longer times, easily visible after the initial exponential decrease. This tail may be due to the release of charge from traps produced by the irradiation. If we assume a single dominant trapping time constant $\tau$ and define

$q_{\mathrm{e}} \quad=$ charge on the electrode;

$q_{\mathrm{e} 0} \quad=$ initial charge on the electrode (from the rapidly collected, untrapped charge);

$q_{\mathrm{to}}=$ initial trapped charge in the cell around the electrode;

$V \quad=$ voltage on the probe;

$V_{0} \quad=$ initial voltage on the probe (from $q_{\mathrm{e} 0} / C$, since at $t=0$, the charge $q_{\mathrm{t} 0}$ is all trapped);

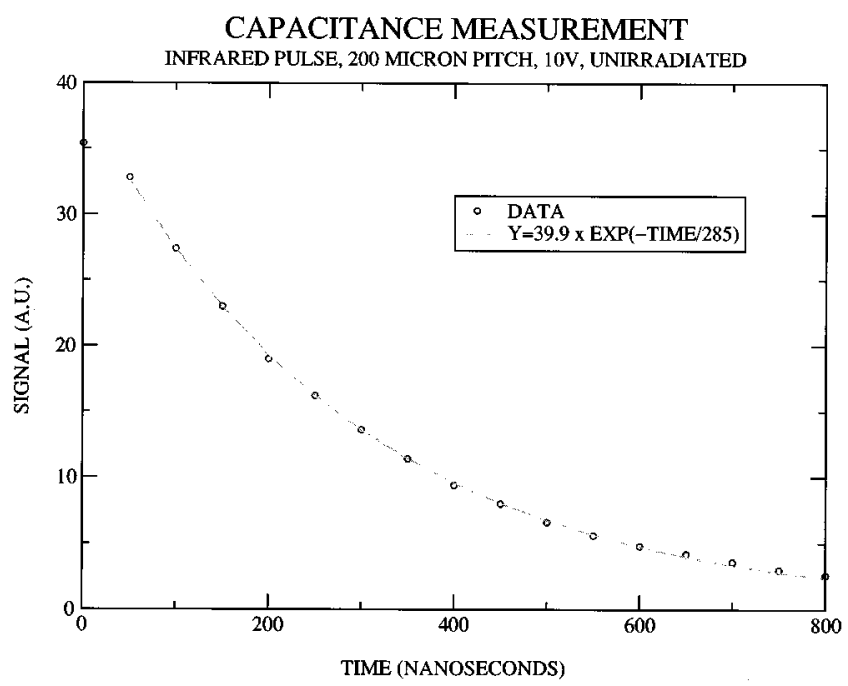

Fig. 6. Decay of the infrared-induced pulse on a p-electrode of an unirradiated $200-\mu \mathrm{m}$ pitch detector with $10 \mathrm{~V}$ bias. The probe has an impedance of $1 \mathrm{M} \Omega$ in parallel with $0.1 \mathrm{pF}$. The light-emitting diode drive pulse is turned off at zero time on the plot. The exponential fit starts with the second point, after the light emission ends and the charge has been collected.

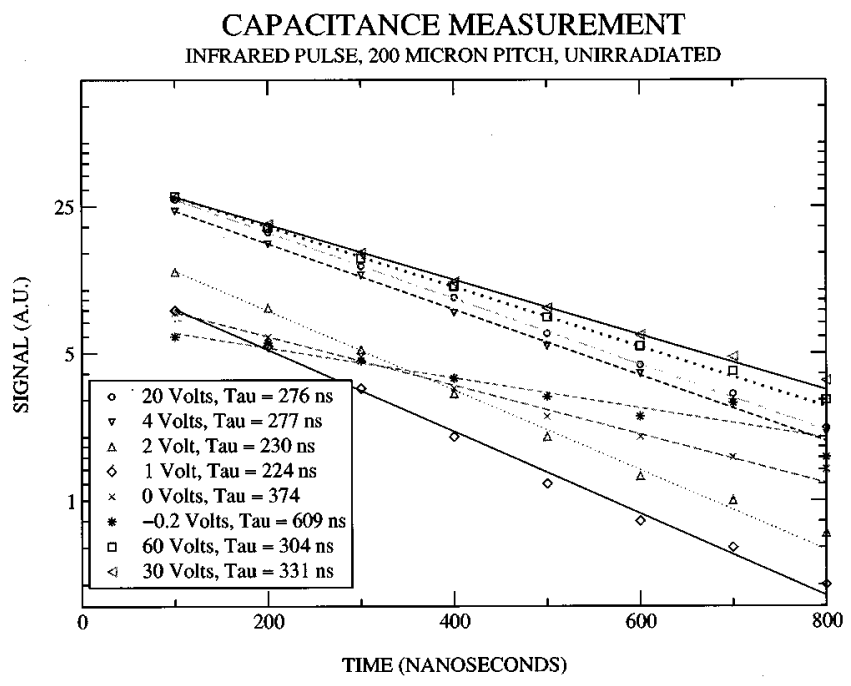

Fig. 7. Decay curves similar to the one in Fig. 6, for bias voltages from 0 to $60 \mathrm{~V}$. 


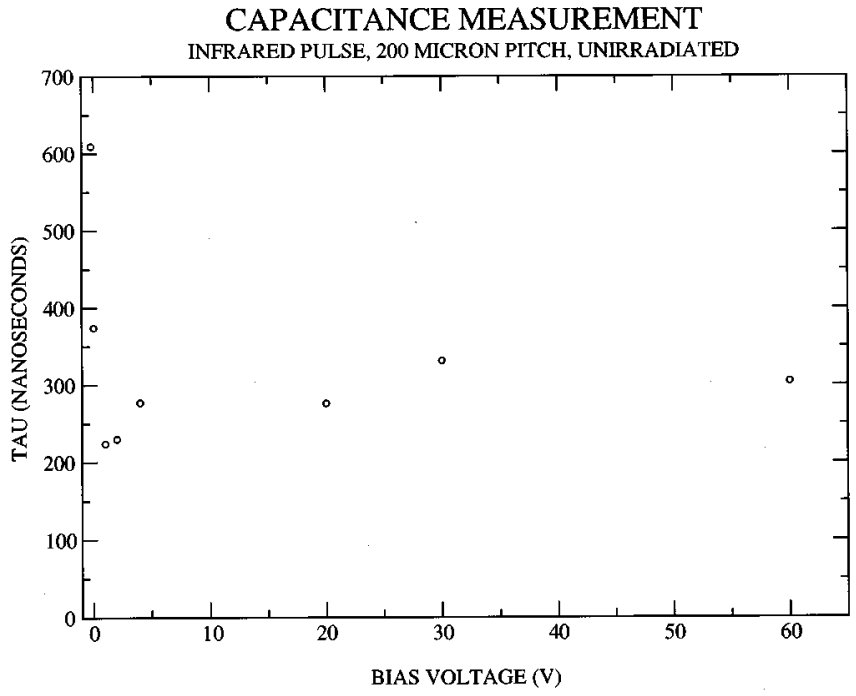

Fig. 8. $R C$ decay times as a function of detector bias voltage, derived from the curves in Figs. 6 and 7.

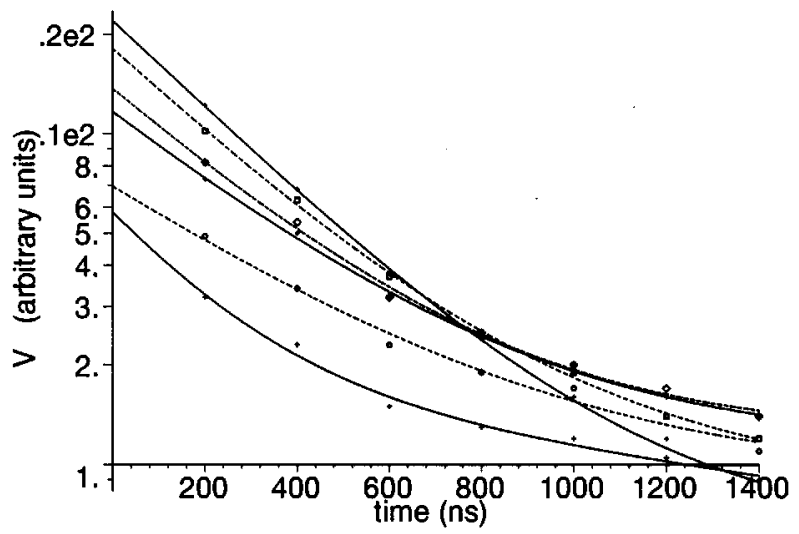

Fig. 9. Decay curves similar to those in Fig. 7 for a detector irradiated with $1 \times 10^{14} 55 \mathrm{MeV}$ protons $/ \mathrm{cm}^{2}$. The curves are fits of (4) to the data points between 200 and $1400 \mathrm{~ns}$, with the curves at the left margin, from highest to lowest, for bias voltages of 45, 30, 20,15, 10, and $5 \mathrm{~V}$.

$V_{\tau 0} \quad=$ voltage the electrode and probe would have if a charge $q_{\mathrm{to} 0}$ were deposited on them;

$R \quad=$ resistance of the probe;

$C \quad=$ capacitance of the electrode and probe.

Then

$$
\begin{aligned}
\frac{d q_{\mathrm{e}}}{d t} & =-I_{\text {to-probe }}+I_{\text {from-traps }} \\
& =-\frac{V}{R}-\frac{d}{d t}\left(q_{\tau 0} \mathrm{e}^{-\frac{t}{\tau}}\right) \\
& =-\frac{q_{\mathrm{e}}}{R C}+\frac{q_{\tau 0}}{\tau} \mathrm{e}^{-\frac{t}{\tau}} .
\end{aligned}
$$

If we divide each term by $C$, we have

$$
\frac{d V}{d t}=-\frac{V}{R C}+\frac{V_{\tau 0}}{\tau} \mathrm{e}^{t / \tau} .
$$

The solution to this equation is

$V=\left[V_{0}-V_{\tau 0}\left(\frac{R C}{\tau-R C}\right)\right] \mathrm{e}^{-\frac{t}{R C}}+V_{\tau 0}\left(\frac{R C}{\tau-R C}\right) \mathrm{e}^{-\frac{t}{\tau}}$

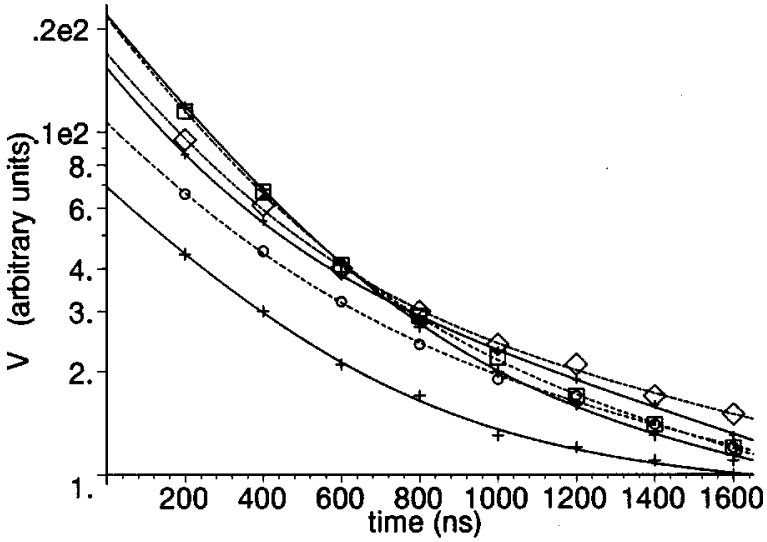

Fig. 10. Decay curves similar to those in Fig. 7 for a detector irradiated with $2 \times 10^{14} 55 \mathrm{MeV}$ protons $/ \mathrm{cm}^{2}$. The curves are fits of (4) to the data points between 200 and $1600 \mathrm{~ns}$, with the curves at the left margin, from highest to lowest, for bias voltages of $60,40,20,15,10$, and $5 \mathrm{~V}$.

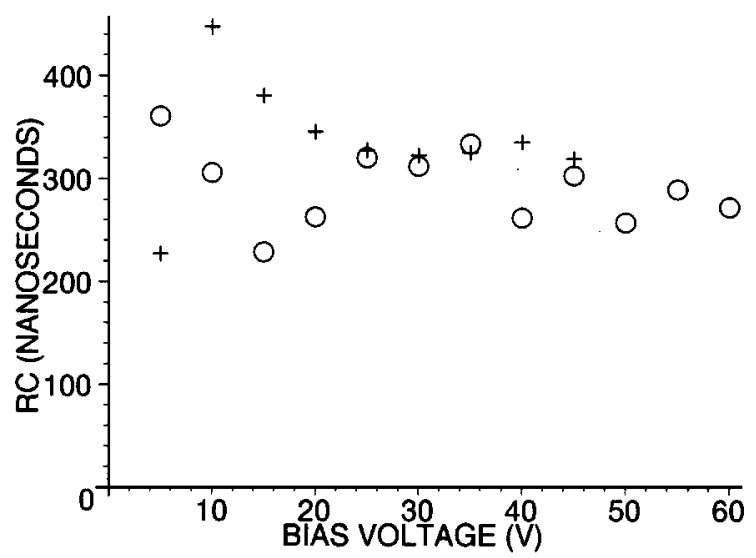

Fig. 11. $R C$ decay times of charge, released by infrared illumination, through a $1 \mathrm{M} \Omega$ picoprobe input resistor, as a function of detector bias voltage. The times are derived from the fits of (4) to the data of Figs. 9 and 10. The 200- $\mu \mathrm{m}$ pitch detectors were irradiated with 1 (crosses) and 2 (circles) $\times 10^{14}, 55$ $\mathrm{MeV}$ protons per $\mathrm{cm}^{2}$.

Fig. 11 shows the $R C$ times derived from those and similar curves for a range of bias voltages for $200-\mu \mathrm{m}$ pitch detectors, irradiated with 1 and $2 \times 10^{14}, 55 \mathrm{MeV}$ protons per $\mathrm{cm}^{2}$. For bias voltages above the full depletion values of 30 and $35 \mathrm{~V}$ for the exposure of 1 and $2 \times 10^{14}$ protons per $\mathrm{cm}^{2}$ [see Fig. 3(b) and (c)], the $R C$ values are 330 and $290 \mathrm{~ns}$, corresponding to a capacitance of $0.2 \mathrm{pF}$. The time constant $\tau$ is an order of magnitude larger, but is not accurately determined due to the limited time range covered for this capacitance measurement and to the smaller size of the signal at those long times. The coefficient of the $\tau$ term is about $10 \%$ of that of the main $R C$ term and for the same reasons is only determined approximately.

\section{CONCLUSION}

Silicon detectors with 3-D electrode arrays have been irradiated with up to $10^{15} 55 \mathrm{MeV}$ protons per $\mathrm{cm}^{2}$ and also with $24 \mathrm{GeV}$ protons. To keep this initial fabrication run simple, no special hardening steps, such as the addition of oxygen to the bulk or of high-resistivity field plates to the field oxide, were taken. The highest dose is equivalent to that expected in over ten years of running, at the 50-mm radius of the Atlas B-layer. After 
this dose, which was limited by the available cyclotron time, a $100-\mu \mathrm{m}$ pitch detector had a $45-\mathrm{V}$-wide plateau that started at about $105 \mathrm{~V}$, over 20 times higher than its 5-V starting point before irradiation. Leakage currents were measured and, for unirradiated devices, had values similar to those measured before [27]. The electrode capacitance was also measured and found to have values close to what would be expected from calculations in the initial publication on 3-D sensors [26].

The low depletion voltages observed here will probably be lowered when oxygen is introduced into the bulk and beneficial annealing is allowed to take place.

\section{ACKNOWLEDGMENT}

The authors would like to thank J. Taylor, the members of the Lawrence Berkeley Laboratory Atlas group, and the staff of the Lawrence Berkeley Laboratory 88-in cyclotron for carrying out the cyclotron irradiation. They would also like to thank C. DaVia for carrying out the detector irradiation at the CERN 24-GeV proton synchrotron and for her continued interest, from the earliest days, in the development of this technology. The fitting of the curves of Figs. 9 and 10 was done with the help of W. Langeveld of the Stanford Linear Accelerator Center.

The authors also appreciate the interest and support of $\mathbf{J}$. Plummer of Stanford University and H. Yamamoto of the University of Hawaii. They have received vital help in the form of computer support and office space from the Stanford Linear Accelerator Center and the Lawrence Berkeley Laboratory. They were also helped in many different ways by the staff of the Stanford Nanofabrication Facility. It was a pleasure to work with them.

\section{REFERENCES}

[1] J. Walker, S. Parker, B. Hyams, and S. Shapiro, "Development of high density readout for silicon strip detectors," Nucl. Instrum. Methods, vol. 226, pp. 200-203, 1984.

[2] H. Boesch Jr and T. Taylor, "Charge and interface state generation in field oxides," IEEE Trans. Nucl. Sci., vol. NS-31, pp. 1273-1279, 1984.

[3] N. Saks and R. Rendell, "The time-dependence of post-irradiation interface trap build-up in deuterium annealed oxides," IEEE Trans. Nucl. Sci., vol. 39, pp. 2220-2229, Dec. 1992.

[4] B. Mrstik, V. Afanas'ev, A. Stesmans, P. McMarr, and R. Lawrence, "Relationship between oxide density and charge trapping in $\mathrm{SiO}_{2}$ films," J. Appl. Phys., vol. 85, pp. 6577-6588, 1999.

[5] D. Pitzl, N. Cartiglia, K. Clark, B. Hubbard, J. Leslie, K. O'Shaughnessy, W. Rowe, H. Sadrozinski, E. Spencer, H. Ziock, P. Ferguson, E. Milner, W. Sommer, and J. Ellison, "Study of radiation effects on AC-coupled silicon strip detectors," Nucl. Phys. B (Proc. Suppl.), vol. 23A, pp. 340-346, 1991.

[6] , More than a hundred papers have been published during the last thirty years on the bulk radiation damage of silicon detectors. Two papers from the early 90 s and three later ones are given below.

[7] F. Lemeilleur, M. Glaser, E. Heijne, P. Jarron, and E. Occelli, "Neutroninduced radiation damage in silicon detectors," IEEE Trans. Nucl. Sci., vol. 39, pp. 551-557, 1992.

[8] K. Gill, G. Hall, S. Roe, S. Sotthibandhu, R. Wheadon, P. Giubellino, and L. Ramello, "Radiation damage by neutrons and photons to silicon detectors," Nucl. Instrum. Methods, vol. A 322, pp. 177-188, 1992.

[9] R. Wunstorf, "Radiation hardness of silicon detectors: Current status," IEEE Trans. Nucl. Sci., vol. 44, pp. 806-814, 1997.

[10] Z. Li, "Systematic modeling and comparisons of capacitance and current-based microscopic defect analysis techniques for measurements of high-resistivity silicon detectors after irradiation," Nucl. Instrum. Methods, vol. A 403, pp. 399-416, 1998.
[11] G. Lindström, M. Moll, and E. Fretwurst, "Radiation hardness of silicon detectors-a challenge from high-energy physics," Nucl. Instrum. Methods, vol. A 426, pp. 1-15, 1999.

[12] H. Ziock, J. Boissevain, K. Holzscheiter, J. Kapustinsky, A. Palounek, W. Sondheim, E. Barberis, N. Cartiglia, J. Leslie, D. Pitzl, W. Rowe, H. Sadrozinski, A. Seiden, E. Spencer, J. Ellison, J. Fleming, S. Jerger, D. Joyce, C. Lietzke, E. Reed, S. Wimpenny, P. Ferguson, M. Frautschi, J. Matthews, and D. Skinner, "Temperature dependence of radiation damage and its annealing in silicon detectors," IEEE Trans. Nucl. Sci., vol. 40, pp. 344-348, 1993.

[13] H. Ziock, K. Holzscheiter, A. Morgan, A. Palounek, J. Ellison, A. Heinson, M. Mason, S. Wimpenny, E. Barberis, N. Cartiglia, A. Grillo, K. O'Shaughnessy, J. Rahn, P. Rinaldi, W. Rowe, H. Sadrozinski, A. Seiden, E. Spencer, A. Webster, R. Wichmann, M. Wilder, M. Frautschi, J. Matthews, D. McDonald, D. Skinner, D. Coupal, and T. Pal, "Temperature dependence of the radiation induced change of depletion voltage in silicon PIN detectors," Nucl. Instrum. Methods, vol. A 342, pp. 96-104, 1994.

[14] E. Fretwurst, H. Feick, M. Glaser, C. Gösslling, E. Heijne, A. Hess, F. Lemeilleur, G. Lindström, K. Mählmann, A. Rolf, T. Schulz, and C. Soave, "Reverse annealing of the effective impurity concentration and long term operational scenario for silicon detectors in future collider experiments," Nucl. Instrum. Methods, vol. A 342, pp. 119-125, 1994.

[15] A. Chilingarov, H. Feick, E. Fretwurst, G. Lindström, S. Roe, and T. Schulz, "Radiation studies and operational projections for silicon in the ATLAS inner detector," Nucl. Instrum. Methods, vol. A 360, pp. 432-437, 1995.

[16] K. Gill, G. Hall, and B. MacEvoy, "Bulk damage effects in irradiated silicon detectors due to clustered divacancies," J. Appl. Phys., vol. 82, pp. 126-136, 1997.

[17] C. Leroy, S. Bates, B. Dezillie, M. Glaser, F. Lemeilleur, and I. Trigger, "Study of charge collection and noise in nonirradiated and irradiated silicon detectors," Nucl. Instrum. Methods, vol. A 388, pp. 289-296, 1997.

[18] E. Borchi, M. Bruzzi, C. Leroy, S. Pirollo, and S. Sciortino, "Charge collection and noise analysis of heavily irradiated silicon detectors," IEEE Trans. Nucl. Sci., vol. 45, pp. 141-145, 1998.

[19] L. Beattie, T. Brodbeck, A. Chilingarov, G. Hughes, P. Ratoff, and T. Sloan, "Charge collection efficiency in heavily irradiated silicon diodes," Nucl. Instrum. Methods, vol. A 412, pp. 238-246, 1998.

[20] L. Beattie, T. Brodbeck, A. Chilingarov, G. Hughes, S. McGarry, P. Ratoff, and T. Sloan, "Carrier lifetimes in heavily irradiated silicon diodes," Nucl. Instrum. Methods, vol. A 421, pp. 502-511, 1999.

[21] B. Dezillie, "Radiation hardness studies of epitaxial silicon particle detectors for applications at the CERN Large Hadron Collider," Ph.D. dissertation, pp. 72-90, 153-162, University Joseph Fourier-Grenoble 1, Grenoble, France, 1997.

[22] V. Augelli, G. Contento, T. Ligonzo, M. Muscarella, L. Schiavulli, M. Angarano, D. Creanza, and M. de Palma, "Electrical characterization of standard and oxygenated irradiated ROSE diodes," Nucl. Instrum. Methods, vol. A 426, pp. 81-86, 1999.

[23] M. Moll, E. Fretwurst, and G. Lindström, "Leakage current of hadron irradiated silicon detectors-material dependence," Nucl. Instrum. Methods, vol. A 426, pp. 87-93, 1999.

[24] A. Ruzin, G. Casse, M. Glaser, and F. Lemeilleur, "Studies of radiation hardness of oxygen enriched silicon detectors," Nucl. Instrum. Methods, vol. A426, pp. 94-98, 1999.

[25] A. Ruzin, G. Casse, M. Glaser, F. Lemeilleur, J. Matheson, S. Watts, and A. Zanet, "Radiation Effects in Silicon Detectors Processed on Carbon and Oxygen Rich Substrates,", CERN RD-48 (ROSE) Collaboration; see also http://hep.ph.liv.ac.uk/ gcasse.

[26] S. Parker, C. Kenney, and J. Segal, "3-D—A proposed new architecture for solid-state radiation detectors," Nucl. Instrum. Methods, vol. A 395, pp. 328-343, 1997.

[27] C. Kenney, S. Parker, J. Segal, and C. Storment, "Silicon detectors with 3-D electrode arrays: Fabrication and initial test results," IEEE Trans. Nucl. Sci., vol. 46, pp. 1224-1236, 1999.

[28] C. Kenney, S. Parker, B. Krieger, B. Ludewigt, T. Dubbs, and H. Sadrozinski, "Observation of beta and X-rays with 3-D-architecture silicon microstrip sensors," IEEE Trans. Nucl. Sci., vol. 48, pp. 189-193, Apr. 2001.

[29] C. Kenney, S. Parker, and E. Walckiers, "Results from 3D silicon sensors with wall electrodes: A preview of active-edge sensors,", Univ. of Hawaii preprint UH 511975 00, 2000.

[30] S. Parker and C. Kenney, "Charge compensation in irradiated semiconductor devices using high-resistivity field plates," Nucl. Instrum. Methods, to be published. 
[31] G. Summers, E. Burke, P. Shapiro, S. Messenger, and R. Walters, "Damage correlations in semiconductors exposed to gamma, electron and proton radiations," IEEE Trans. Nucl. Sci., vol. 40, pp. 1372-1379, 1993.

[32] M. Huhtinen and P. Aarnio, "Pion induced displacement damage in silicon devices," Nucl. Instrum. Methods, vol. A 335, pp. 580-582, 1993.

[33] S. Bates, C. Furetta, M. Glaser, F. Lemeilleur, C. Soave, and E. LeónFlorián, "Damage induced by pions in silicon detectors," Nucl. Phys. B (Proc. Suppl.), vol. 44, pp. 510-519, 1995.

[34] U. Biggeri, E. Borchi, M. Bruzzi, A. Candelori, and A. Giraldo, "Comparison of radiation damage in silicon detectors induced by pions, protons and neutrons," Il Nuovo Cimento, vol. 109 A, pp. 1351-1358, 1996.

[35] H. Feick, E. Fretwurst, G. Lindström, and M. Moll, "Long term damage studies using silicon detectors fabricated from different starting materials and irradiated with neutrons, protons and pions," Nucl. Instrum. Methods, vol. A 377, pp. 217-223, 1996.

[36] S. Bates, C. Furetta, M. Glaser, F. Lemeilleur, E. León-Florián, C. Gössling, B. Kaiser, A. Rolf, R. Wunstorf, H. Feick, E. Fretwurst, G. Lindström, M. Moll, G. Taylor, and A. Chilingarov, "Pion-induced damage in silicon detectors," Nucl. Instrum. Methods, vol. A 379, pp. 116-123, 1996.
[37] I. Lazanu, S. Lazanu, U. Biggeri, E. Borchi, and M. Bruzzi, "Non-ionising energy loss of pions in thin silicon samples," Nucl. Instrum. Methods, vol. A 388, pp. 370-374, 1997.

[38] A. Ruzin, G. Casse, M. Glaser, A. Zanet, F. Lemeilleur, and S. Watts, "Commparison of radiation damage in silicon induced by proton and neutron irradiation," IEEE Trans. Nucl. Sci., vol. 46, pp. 1310-13, 1999.

[39] P. Aarnio and M. Huhtinen, "Hadron fluxes in inner parts of LHC detectors," Nucl. Instrum. Methods, vol. A 336, pp. 98-105, 1993.

[40] V. Cindro, G. Kramberger, M. Mikuž, and D. žontar, "Bias-dependent annealing of radiation damage in neutron-irradiated silicon $\mathrm{p}^{+}-\mathrm{n}-\mathrm{n}^{+}$ diodes," Nucl. Instrum. Methods, vol. A 419, pp. 132-136, 1998

[41] V. Cindro, G. Kramberger, M. Mikuž, M. Tadel, and D. Žontar, "Biasdependent radiation damage in high-resistivity silicon diodes irradiated with heavy charged particles," Elsevier Preprint, Atlas Preprint ATLINDET 99-010, Aug. 1999, submitted for publication. 